\title{
Proximal Type Epithelioid Sarcoma Presenting as Large Multinodular Peritoneal Mass
}

Sunayana Misra*, Chhagan Bihari Sharma, Archana Rastogi, Rajesh S and Dipanjan Panda

ILBS, Pathology, D1, Vasant Kunj, Delhi 110070, India

*Corresponding author: Sunayana Misra, PDCC Senior Resident, ILBS, Pathology, D1, Vasant Kunj, Delhi 110070, India, Tel: 918376881696; E-mail: sunayanamisra@gmail.com

Rec date: Feb 10, 2015, Acc date: Feb 28, 2015, Pub date: Mar 02, 2015

Copyright: $\odot 2015$ Misra S, et al. This is an open-access article distributed under the terms of the Creative Commons Attribution License, which permits unrestricted use, distribution, and reproduction in any medium, provided the original author and source are credited.

\begin{abstract}
Proximal type of epithelioid sarcoma is a rare variant which arises in deep locations such as pelvis and perineal soft tissue. It affects the middle aged to elderly and is more commonly seen in males. This entity needs to be recognized as it has a wide spectrum of differential diagnosis. Here, we discuss a case of proximal epithelioid sarcoma which presented as a large intraperitoneal multinodular mass with a radiologic impression of peritoneal sarcomatosis.
\end{abstract}

Keywords: Proximal epithelioid sarcoma; Intraperitoneal metastasis; Multidetector computed tomographic scan

\section{Introduction}

Epithelioid sarcoma is a rare tumor which was first described by Enzinger in 1970 [1]. The classic variant primarily affects young adults, more commonly females and usually arises in distal extremities. A proximal variant of epithelioid sarcoma, which was first described by Guillou in 1997, demonstrates a more aggressive clinical course [2]. Proximal type of epithelioid sarcoma affects middle-aged patients and arises in deep seated locations, primarily the pelvis, perineum and the genital tract [2]. It usually presents as a poorly circumscribed painful mass lesion. It is important to recognize this entity, especially on small biopsies, where it may be mistaken for benign conditions due to its necrotizing granuloma like pattern or undifferentiated metastatic carcinoma due to its epithelial look and positivity for cytokeratin/ epithelial membrane antigen (EMA).

\section{Case Presentation}

A 38 year old man presented with heaviness and pain in abdomen since four months. There was no history of trauma, fever or weight loss. Liver function tests were found to be within normal limits except for mildly increased alkaline phosphatase. Serum Lactate dehydrogenase level was found to be increased. Tumor marker levels for carcino-embryonic antigen, alpha-feto protein and CA 19-9 were found to be within the normal range.

The patient underwent multidetector computed tomographic (MDCT) scan of the abdomen and pelvis on a Discovery 750 HD 64row spectral CT scanner (General Electric, Wisconsin, USA); the helical scan parameters included: $120 \mathrm{kV}$ with automated $\mathrm{mA}$, pitch of 1.375:1, detector coverage of 40-mm and matrix size of $512 \times 512$. Approximately $100 \mathrm{ml}$ of low osmolarity non-ionic contrast media (Iomeron; 1.5 to $2.0 \mathrm{~mL} / \mathrm{kg}$ bodyweight, $400 \mathrm{mg} / \mathrm{mL}$ ) was administered intravenously at a rate of $3.0-3.5 \mathrm{~mL} / \mathrm{s}$ and scan obtained in the arterial, portal venous and equilibrium phases with a delay of 25 $\mathrm{s}, 60 \mathrm{~s}$ and $180 \mathrm{~s}$, respectively, with a slice of $2.5 \mathrm{~mm}$ thickness. MDCT revealed multiple bulky, lobulated, intraperitoneal soft tissue masses of varying sizes ranging from $4 \times 3 \times 3 \mathrm{~cm}$ to $15 \times 8.5 \times 6.5 \mathrm{~cm}$. These masses were found to be highly vascular with rich arterial networks and areas of arterio-venous shunting on the CT scan. The average attenuation of the lesions ranged from $40-50 \mathrm{HU}$ on pre-contrast scan which increased to $120-140 \mathrm{HU}$ on the arterial phase images. Diffuse infiltration of the inguinal canal as well as gastro-hepatic ligament, left hemidiapharagm, hepatic segments II/III and caudate lobe were noted. There was no organomegaly; however gross ascites and thickening of the parietal peritoneum were noted. A radiological impression of peritoneal sarcomatosis was given (Figure 1). Ultrasound guided core biopsies were taken from the pelvic and left flank masses. MDCT of chest was performed to rule out thoracic involvement and it showed nodular deposits along the sub-diaphragmatic aspect of the left hemidiaphragm with possible contiguous intrathoracic extension (Figure 2). Mediastinal structures, lungs and tracheobronchial tree were found to be normal.

On microscopy, the biopsy showed lobules of tumor separated by fibrous septae. Within the nodules, tumor was arranged predominantly in sheets and focally as dissociated singly scattered cells. The tumor cells were moderately pleomorphic, polygonal in shape with large nuclei, opened up chromatin, prominent nucleoli and moderate to copious amounts of eosinophilic cytoplasm. Few of the cells had eccentric nuclei and intracytoplasmic hyaline inclusions giving a rhabdoid appearance. Areas of necrosis were identified along with a few scattered mitotic figures were seen. Surrounding inflammation composed of lymphoplasmaytic cells along with a few neutrophils was noted (Figure 3).

A wide panel of immunohistochemical markers was applied to characterize the tumor. The tumor cells were strongly positive for EMA, CK 19 and vimentin, however, CD 34 was found to be negative. In addition, tumor was negative for S-100, Arginase, Hep Par 1, CD 68, CD 99 and HMB 45. Desmin was found to be focally positive in the 
Citation: Misra S, Sharma CB, Rastogi A, Rajesh S, Panda D (2014) Proximal Type Epithelioid Sarcoma Presenting as Large Multinodular Peritoneal Mass. J Cytol Histol S3: 002. doi:10.4172/2157-7099.S3-002

Page 2 of 3

larger rhabdoid cells (Figure 4). Both internal and external controls (normal skeletal muscle, endothelial cells, nerves and liver) reacted as expected.

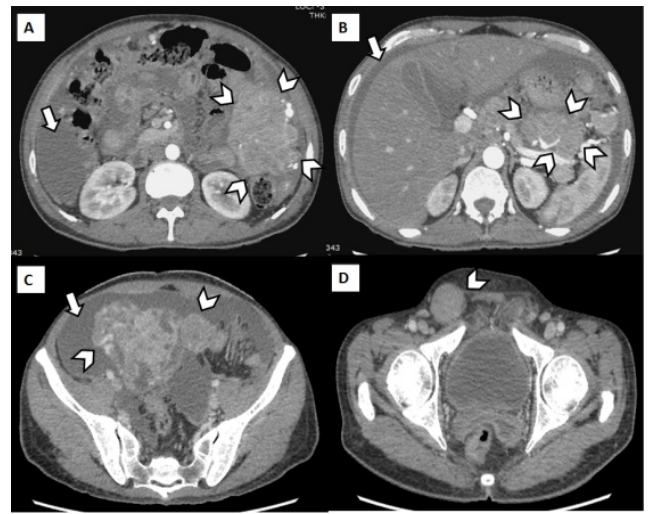

Figure 1: Axial MDCT scan images of the abdomen acquired in the arterial $(\mathrm{A}, \mathrm{B})$ and venous phases $(\mathrm{C}, \mathrm{D})$ demonstrating multiple bulky, lobulated, heterogeneously enhancing, intraperitoneal soft tissue masses in the left para-colic gutter, lesser sac and pelvis (arrowheads in A, B and C, respectively) with moderate ascites (arrows). Similar soft-tissue lesions were seen diffusely scattered in the entire peritoneal cavity (not shown). Associated nodular thickening of the parietal peritoneum was present which was extending into the right inguinal canal (arrowhead in D).

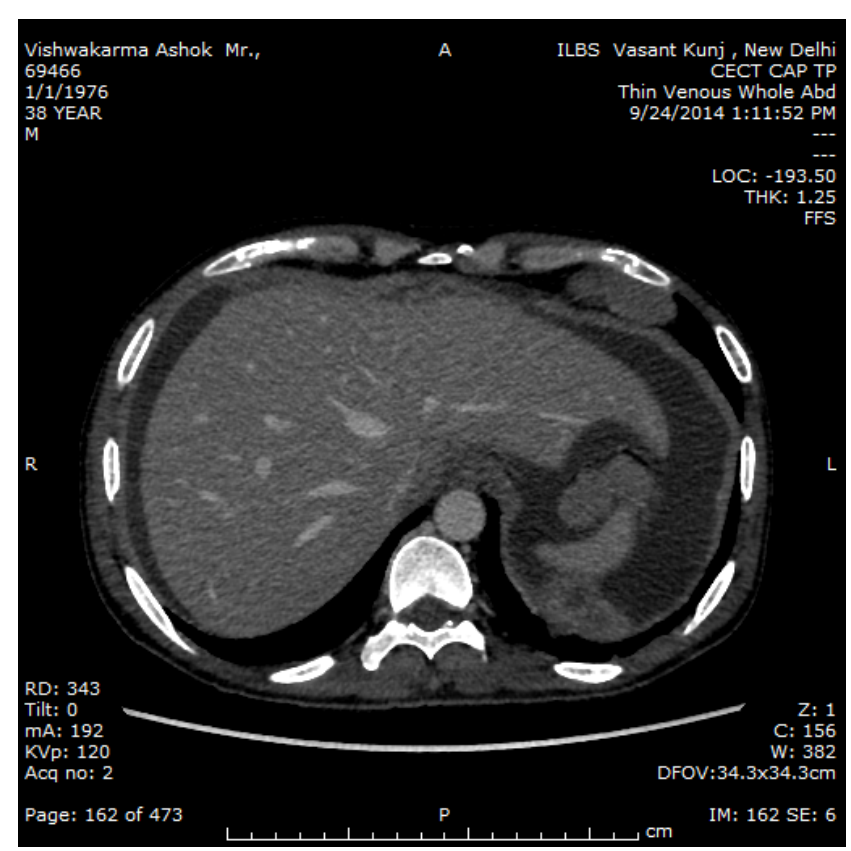

Figure 2: MDCT scan of the abdomen (mediastinal window) demonstrating left diaphragmatic lobulated mass with slight encasement of pleural compartment.

\section{Discussion}

The clinical, histological and immunohistochemical features of the present case are in accordance with that of proximal type of epithelioid sarcoma. Our case presented as multiple peritoneal nodules, which has not been reported previously as an initial presentation. Guillou et al, in their case series of proximal epithelioid sarcomas, described six cases with perineal or pubic masses [2]. Rekhi et al. described a case with recurrent perineal tumor [3]. Other studies have reported these tumours to be arising in the vulva, thigh, deep soft tissue and buttocks [2-5].

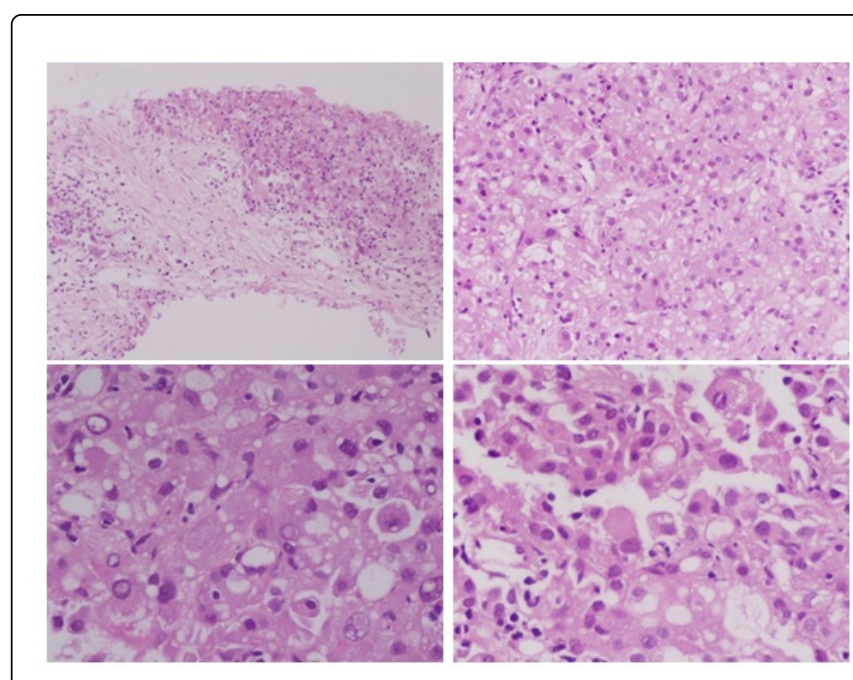

Figure 3: (A) Low power photomicrograph of the core biopsy showing nodules of tumor cells separated by fibrous septae $(\mathrm{H}$ and E, 10x). (B) Sheet like arrangement of tumor cells within the lobules ( $\mathrm{H}$ and $\mathrm{E}, 20 \mathrm{x})$. (C) Higher magnification showing large polygonal tumor cells with abundant cytoplasm and nuclear pseudoinclusions (H and E, 40x). (D) Rhabdoid morphology of tumor cells ( $\mathrm{H}$ and $\mathrm{E}, 40 \mathrm{x})$.
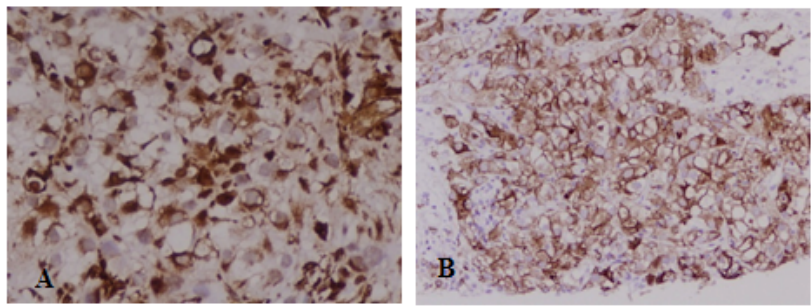

Figure 4: (A) Immunohistochemistry showing strong, diffuse cytoplasmic staining for Vimentin and (B) strong membranous staining for EMA.

These tumors are seen in middle-aged individuals and have a significant male predominance. On histopathology, they usually lack the "granuloma-like appearance" of conventional epithelioid sarcomas and are characterized by prominence of epithelioid cytomorphology, sheet like growth pattern of large cells with vesicular nuclei, prominent nucleoli and frequent occurrence of rhabdoid features. Immunohistochemistry is positive for cytokeratins, EMA and vimentin. CD 34 positivity is seen in about half of the cases [2]. 
Proximal type of epithelioid sarcoma has a wide range of differentials including metastasis from a poorly differentiated carcinoma, malignant extra renal rhabdoid tumors (MERT), epithelioid leiomyosarcoma, epithelioid gastro-intestinal stromal tumour (GIST), epithelioid malignant peripheral nerve sheath tumour (MPNST), rhabdomyosarcoma, synovial sarcoma, mesothelioma and anaplastic large cell lymphoma (ALCL) [3]. Contrast enhanced magnetic resonance imaging (MRI) is widely accepted as the imaging modality of choice for investigating a suspected case of peritoneal sarcomatosis due to its superior contrast resolution and ability to depict different inherent characteristics of the disease process by acquiring multiple image sequences. However, the case presented here came to us with non-specific complaints and CECT was considered to be an appropriate initial imaging investigation. Moreover, since there is a significant overlap in imaging features of soft tissue sarcomas, the final diagnosis and management depends on histopathology and immunohistochemistry. Role of imaging is primarily to determine the exact location of the lesion, its extent and involvement of adjacent structures and also to rule out metastases. Hence, an MRI was not carried out in our case.

To sort out the wide list of differentials, narrow it down and to reach a single diagnosis which is in accordance with the clinicoradiologic findings, we took the help of immunohistochemistry. Since there was no mass lesion detected in the visceral organs during metastatic workup and tumor marker levels were normal, the possibility of metastasis from a primary in the gastrointestinal tract, genitourinary tract or lungs were ruled out. Lack of LCA, S-100 and HMB-45 ruled out the possibility of ALCL, epithelioid MPNST and malignant melanoma respectively. Though desmin was focally positive in the larger cells, rhabdomyosarcoma was excluded as the tumor cells showed complete, strong, membranous staining with EMA and cytokeratin 19. There was no biphasic pattern in any of the biopsies to favour a diagnosis of synovial sarcoma; also CD 99 was found to be negative.

The pathognomonic feature of MERT is the presence of rhabdoid cells with intracytoplasmic inclusions, which prove ultrastructurally to be whorls of intermediate filaments [3]. This tumor type has been documented in a variety of sarcomas and also in carcinomas and melanomas. Its distinction from proximal-type epithelioid sarcoma may be difficult at times. Extrarenal rhabdoid tumor, like the renal counterpart, is a highly aggressive and lethal neoplasm occurring in younger children and has inactivating mutations or deletions of both alleles of the tumor suppressor gene, hSNF5/INI1 on chromosome 22q11.2 [6]. However, alterations in MERT are more frequently point mutations, not deletions as seen in epithelioid sarcoma [6]. Also, it does not correlate with the variety of morphologies seen in epithelioid sarcoma.

The management protocol for patients with epithelioid sarcoma in absence of metastases is wide local excision with resection of tumor margins. After surgery, chemotherapy and radiotherapy is also advocated due to high incidence of local recurrence and distant metastases. Prognosis of proximal-type epithelioid sarcoma depends on tumor size and presence of rhabdoid features [3]. Rhabdoid features indicate aggressive behavior, multimodality therapy resistance and rapidly fatal outcome [3]. Thus, it is important to diagnose this rare variety of soft tissue sarcoma as it is a rapidly growing, aggressive tumor. Due to the inconsistent pathologic features of these tumors, it is of utmost importance to recognize this entity, especially on small biopsy specimen so that a proper treatment can be offered to the patient, if detected early.

\section{References}

1. Enzinger FM (1970) Epitheloid sarcoma. A sarcoma simulating a granuloma or a carcinoma. Cancer 26: 1029-1041.

2. Guillou L, Wadden C, Coindre JM, Krausz T, Fletcher CD (1997) "Proximal-type" epithelioid sarcoma, a distinctive aggressive neoplasm showing rhabdoid features. Clinicopathologic, immunohistochemical, and ultrastructural study of a series. Am J Surg Pathol 21: 130-146.

3. Rekhi B, Gorad BD, Chinoy RF (2007) Proximal-type epithelioid sarcoma--a rare, aggressive subtype of epithelioid sarcoma presenting as a recurrent perineal mass in a middle-aged male. World J Surg Oncol 5: 28.

4. Naik D, Kumar AA, Srinath MG (2011) Proximal-type epithelioid sarcoma-a rare soft tissue sarcoma of thigh in a child. Indian J Surg Oncol 2: 298-301.

5. Ulbright TM, Brokaw SA, Stehman FB, Roth LM (1983) Epithelioid sarcoma of the vulva. Evidence suggesting a more aggressive behavior than extra-genital epithelioid sarcoma. Cancer 52: 1462-1469.

6. Hornick JL, Dal Cin P, Fletcher CD (2009) Loss of INI1 expression is characteristic of both conventional and proximal-type epithelioid sarcoma. Am J Surg Pathol 33: 542-550. 